\author{
Pawet Matyaszewski \\ http:/orcid.org/0000-0001-6214-6871 \\ Université catholique de Lublin Jean-Paul II \\ pawelm@kul.pl

\section{LES TRADUCTIONS \\ POLONAISES DES LETTRES \\ PERSANES \\ DE MONTESQUIEU}

Polish translations of Montesquieu's Persian Letters

\begin{abstract}
The purpose of the present article is to show the history of Polish translations of a famous epistolary novel Persian Letters written in 1721 by Montesquieu. In his work, the author neither introduces linguistic matters of the translations, nor he analyses their correctness; however, he presents strictly historical and cultural aspects of the process of translation. What is more, the article aims at presenting the intentions of Polish translators, the historical context of their work and its importance for a Polish reader. Regardless of the era when they produced their translations, starting as early as the eighteenth century, Polish translators always saw in Montesquieu's Persian Letters something more than a simple and light epistolary novel of the Regency era.
\end{abstract}

KEYWORDS: Montesquieu, Persian Letters, Polish translations, Tomasz Kajetan Węgierski, Tadeusz BoyŻeleński.

Tout porte à croire que le plus célèbre roman de Montesquieu ne se fera connaître en Pologne qu'après la mort de l'auteur, durant la seconde moitié du XVIII ${ }^{\mathrm{e}}$ siècle. On sait (Smoleński 1927 : 50) qu'un exemplaire de la toute première édition des Lettres persanes, publiée à Amsterdam en 1721, se trouve à Varsovie dans le fond de la bibliothèque collégiale des pères piaristes, fondateurs du fameux Collegium Nobilium, établissement scolaire moderne, dont les activités pédagogiques contribuent fort à l'essor de l'esprit éclairé en Pologne. Mieux encore, en 1760, l'un d'eux, Stanisław Konarski, écrivain, enseignant et penseur politique célèbre, rappelle avec enthousiasme le roman de Montesquieu dans son fameux ouvrage, O skutecznym rad sposobie (Sur l'organisation efficace des conseils). Le piariste polonais y met en valeur et glorifie la sagesse politique que l'on peut tirer de l'histoire de l'État des Troglodytes, qu'il trouve digne de la République de Platon, de l'Utopie de Thomas More, et de ce qu'il appelle le Royaume d'Eldorado de Voltaire (Konarski 1760 : 51). Ainsi Konarski est-il le premier dans la Pologne de l'époque des Lumières non seulement à parler favorablement des Lettres persanes de Montesquieu, mais aussi, sinon surtout, à y découvrir plus qu'un simple roman épistolaire.

C'est sans aucun doute l'édition posthume des Euvres complètes de Montesquieu, parue en trois volumes à Paris, en 1758, supervisée par son fils, Jean-Baptiste 
de Montesquieu, qui fera mieux connaître, auprès du public polonais, aussi bien les Lettres persanes que d'autres ouvrages majeurs de l'auteur français. C'est aussi à partir de cette édition que verront le jour les toutes premières traductions polonaises de Montesquieu, à commencer par les Considérations sur les causes de la grandeur des Romains et de leur décadence, traduites par le père piariste Antoni Wiśniewski en 1762, et L'Esprit des lois, rendu accessible au lecteur polonais, en 1777, grâce à Mateusz Czarnek, juriste et secrétaire du roi de Pologne, Stanislas-Auguste Poniatowski. Le fait que l'on connaît aujourd'hui les noms des deux traducteurs polonais ne doit pourtant tromper personne : c'est sous l'anonymat que toutes les versions polonaises des ouvrages de Montesquieu paraîtront tout au long du XVIII siècle. Selon l'usage de l'époque, le traducteur tient à s'effacer complètement en faveur de l'auteur, afin de mettre en valeur le nom de l'écrivain et de passer sous silence le sien (Matyaszewski 2013 : 164).

Tel est aussi le cas de la toute première tentative pour traduire en polonais les Lettres persanes, à cette différence près qu'il ne s'agit que d'un tout petit échantillon de tout le roman et que, qui pis est, le traducteur rend anonyme non seulement son propre nom, mais aussi celui de Montesquieu, jusqu'à faire disparaître le titre même de l'ouvrage. En 1767, dans le numéro 94 de l'hebdomadaire Monitor (Moniteur), périodique littéraire et politique important dans l'histoire des Lumières en Pologne, inspiré directement de l'anglais The Spectator, on tombe, sans s'en rendre compte, sur une traduction de deux lettres du roman de Montesquieu (Monitor 1767 : 795-801). Il s'agit de la lettre LII présentée en entier et de la lettre CX légèrement modifiée, les deux mises ensemble et formant un tout, de sorte que le lecteur polonais a l'impression de lire un seul texte, voire une longue lettre. Cette dernière, adressée au rédacteur de Monitor et signée du nom d'un certain Wodnicki, fait semblant d'être une observation ironique portant sur les mœurs des femmes du monde. À vrai dire, dans son jeu de déguisement, le traducteur polonais anonyme « emprunte » directement aux deux lettres persanes de Montesquieu, où Rica regarde et commente les habitudes des Parisiennes avec une grande dose d'étonnement et de moquerie.

Si le ton plutôt léger de ces deux fragments des Lettres persanes ne semble pas correspondre au caractère sérieux du périodique polonais, il n'en est pas de même avec la traduction d'une autre lettre du roman que l'on peut y découvrir deux ans plus tard. En 1769, dans le numéro 54 de Monitor, on révèle une lettre adressée à son rédacteur, où un certain Nieukolubski, en vrai esprit rousseauiste, met en doute les bienfaits des sciences et avertit des dangers de la civilisation (Monitor 1769 : 432-437). Le travestissement épistolaire ne sait pourtant pas duper le lecteur, du moins celui qui connaît bien le roman de Montesquieu, et ne l'empêche guère d'y reconnaître presque toute la lettre LV, un peu remaniée, que Rhédi a envoyée à Usbek. Malheureusement, la très sage réponse que ce dernier donne aussitôt à son ami persan dans la lettre LVI, où il vante l'essor des sciences et le bonheur du progrès de la civilisation humaine, n'a pas été traduite par l'auteur polonais. Faute de place ? - il ne faut pas oublier que la lettre d'Usbek est deux fois plus longue que celle de Rhédi. Le traducteur polonais préfère la remplacer par une courte réplique du rédacteur de Monitor, où celui-ci défend le primat du savoir et l'importance des découvertes scientifiques (Monitor 1769 : 437-438). Finalement, il exprime exactement le même esprit de l'homme des Lumières que le héros persan, voire soutient la profession de foi philosophique de Montesquieu. 
Il est plutôt difficile, sinon quasiment impossible, de dévoiler avec certitude l'identité du traducteur polonais anonyme. On croit qu'il s'agirait de Jan Chrzciciel Albertrandi, jésuite, homme de lettres, poète, représentant éminent des Lumières polonaises (Aleksandrowska 1988 : 157). L'hypothèse n'est pas à exclure, d'autant plus qu'en 1770 Albertrandi fait publier, sous l'anonymat, sa traduction du Dialogue de Sylla et d'Eucrate de Montesquieu, insérée dans Zabawy przyjemne i pożyteczne (Jeux agréables et utiles), hebdomadaire littéraire important, édité à Varsovie dans un milieu intellectuel proche de Monitor. Quoiqu'il soit capital de savoir un jour qui se cache réellement derrière la traduction des trois lettres du roman de Montesquieu, il ne l'est pas moins de comprendre sa décision de passer sous silence le titre et le nom de l'auteur des Lettres persanes. Contrairement à ce que l'on pourrait croire au premier abord, il ne s'agit guère de plagier Montesquieu, mais plutôt de le sauvegarder contre la censure ecclésiale de l'époque. Il ne faut pas oublier que, dès leur apparition, les Lettres persanes sont accusées, d'abord en France et puis en Pologne, d'être impies et blasphématoires, hérétiques et contraires aux dogmes de la religion catholique. On doit rappeler qu'en 1762, l'Index condamne l'ouvrage comme indécent et contraire aux leçons de l'Église (Volpilhac-Auger 2017 : 272), ce qui ne fait qu'aggraver la situation. La réprobation qu'encourent les Lettres persanes, ainsi que l'anathème qui pèse sur elles, permettent de comprendre la décision des rédacteurs de Monitor de ne pas révéler le nom de Montesquieu, ni même de suggérer qu'il s'agit de la traduction d'un fragment de son roman. Cette dernière prouve pourtant que l'on cherche alors en Pologne à propager l'ouvrage, afin de le faire mieux connaître auprès du public polonais éclairé.

En effet, en 1778 paraît à Dresde, en Saxe, en deux volumes successifs, une traduction polonaise complète du roman de Montesquieu. Conformément à l'usage, le nom du traducteur reste anonyme, mais on découvre assez vite qu'il s'agit de Tomasz Kajetan Węgierski, homme de lettres, poète, voyageur, franc-maçon, traducteur de quelques auteurs français de son époque, tels Voltaire, Rousseau ou Marmontel. Sa traduction des Lettres persanes doit sans doute jouir d'un grand succès et d'une grande estime auprès des lecteurs polonais, puisqu'en 1785 et en 1804 on note ses deux rééditions, toujours chez le même éditeur en Saxe et aussi en deux volumes. Dans son travail, Węgierski s'appuie sur la version du roman contenue dans les Euvres complètes de Montesquieu de 1758, mentionnée déjà plus haut (contrairement à l'édition princeps de 1721 qui comporte 150 lettres, elle en possède 161 -c'est elle qui servira habituellement de texte de base à toutes les éditions postérieures du roman). Węgierski traduit aussi tous les éclaircissements et commentaires faits par Montesquieu en bas de pages, et dont la fonction était d'expliquer au lecteur français, voire européen, certaines nuances et caractéristiques de la culture persane, voire orientale. Il rappelle aussi l'Introduction rédigée de la main de Montesquieu, qui, depuis l'édition de 1721, accompagne le roman et où, conformément à la mode de l'époque, l'auteur joue avec le lecteur et passe faussement pour le traducteur des lettres présumées authentiques, afin de garantir à son ouvrage une apparence de véridicité. Curieusement, Węgierski néglige pourtant les Quelques réflexions sur les "Lettres persanes », paratexte qui paraît pour la première fois dans l'édition des Euvres complètes de Montesquieu de 1758. De plus, le traducteur polonais s'abstient de proposer une note critique explicative, ou de faire dans son travail un commentaire personnel quelconque. Il n'intervient directement qu'une seule 
fois, sous forme d'un petit éclaircissement en bas de page adressé au lecteur à propos de la fameuse, et combien controversée lettre XXIV, dans laquelle Montesquieu se moque de la majesté du pape et ironise sur le dogme de la Trinité et sur l'Eucharistie. Afin d'atténuer le ton virulent de ce fragment du roman, ainsi que pour faire éviter, aussi bien à l'auteur français qu'à lui-même, toute accusation d'impiété, Węgierski justifie la moquerie par la différence des cultures et des religions entre l'Europe et l'Orient, voire par l'ignorance du monde chrétien de la part des voyageurs persans (voll. I : 75). Curieusement donc, il se sert exactement du même type d'argumentation, voire d'un stratagème astucieux similaire, que l'on voit dans les Quelques réflexions sur les "Lettres persanes », où leur auteur estime qu' " en parlant de notre religion, ces Persans ne devaient pas paraître plus instruits que lorsqu'ils parlaient de nos coutumes et de nos usages » (Montesquieu 1964 : 22). Sans avoir donc traduit le commentaire explicatif qui se trouve dans les Euvres complètes de Montesquieu de 1758, Węgierski en préserve pourtant l'idée et, heureusement, se refuse ainsi à enlever ou, du moins, à censurer la fameuse lettre XXIV. Tout comme l'auteur des Lettres persanes, le traducteur polonais préfère, en homme des Lumières qu'il est et au nom de la liberté d'expression qu'il professe, s'exposer à des accusations ou attaques possibles plutôt que modifier le texte et toucher à son intégralité.

Il faudra attendre plus d'un siècle pour voir paraître une nouvelle traduction polonaise des Lettres persanes. On sait qu'entre temps la situation géopolitique de la Pologne a radicalement changé : victime des trois partages effectués successivement par les pays voisins, la Russie, la Prusse et l'Autriche, le Royaume de Pologne disparaît définitivement de la carte d'Europe en 1795. Sans vouloir entrer ici plus en détail, il faut rappeler que la nouvelle situation dans laquelle se trouve la Pologne qui, tout simplement, n'existe pas en tant qu'État libre et indépendant, n'est pas propice à propager les idées de Montesquieu, et encore moins à les traduire en polonais. Contrairement à l'énorme intérêt que l'on porte au philosophe français au XVIII ${ }^{\mathrm{e}}$ siècle (Lukowski 2001 : 4959), chez qui on cherche des leçons de sagesse et des conseils pratiques, utiles dans les tentatives de réformes d'un pays en crise sociale et politique alarmante, les Polonais du siècle suivant s'intéressent peu à l'héritage intellectuel de l'auteur de l'Esprit des lois. Leur attitude apparemment étonnante se laisse pourtant expliquer aisément : si, à l'époque des Lumières, Montesquieu doit montrer comment remédier à la situation désespérante d'une Pologne en difficulté, la question cesse, faute d'État, d'être pertinente tout au long $\mathrm{du} \mathrm{XIX}^{\mathrm{e}}$ siècle. Les Polonais cherchent alors la réponse plutôt à la question de savoir par quels moyens reconquérir leur liberté et non comment l'organiser, de quelle façon faire renaître leur pays et non comment le réformer, ce qui fait que, à tort ou à raison, c'est avec moins d'enthousiasme qu'auparavant qu'ils lisent les ouvrages de Montesquieu ou les rendent plus proches des lecteurs polonais (Matyaszewski 2018 : 77-78; 115-116).

C'est donc avec une certaine surprise qu'il faut saluer au $\mathrm{XIX}^{\mathrm{e}}$ siècle l'apparition d'une nouvelle traduction polonaise des Lettres persanes. Elle vit le jour à Varsovie, alors sous le joug russe, en 1885, en un volume, signée des initiales $M$.W. Curieusement, le traducteur dont l'identité n'est toujours pas connue jusqu'aujourd'hui, renoue avec la tradition de l'époque des Lumières et laisse son ouvrage anonyme, sans qu'il soit possible de connaître les raisons de sa décision. Qui pis est, il ne rappelle pas la traduction de Węgierski - on ne sait pas s'il la passait sous silence volontairement ou, 
au contraire, s'il ignorait tout simplement son existence. Tout comme son prédécesseur du XVIII ${ }^{e}$ siècle, le traducteur anonyme ne propose aucune introduction à son ouvrage, ce qui ne l'empêche pourtant d'intervenir parfois dans le texte par des commentaires explicatifs. Or, il ne se limite pas à rappeler les notes de Montesquieu de bas de pages, mais les modifie parfois selon ses désirs. Par exemple, lorsque le romancier écrit dans la lettre XIX qu' « une poignée de Chrétiens sortis d'un rocher font suer les Ottomans et fatiguent leur empire » (Montesquieu $1964: 50$ ), le traducteur remarque qu'il s'agit de l'île de Malte (p. 36) ; en insistant ainsi sur la topographie, il remanie legèrement le commentaire de Montesquieu, d'après lequel « ce sont apparemment les chevaliers de Malte ». Ensuite, à propos de la lettre LXXVIII où, en parlant des Espagnols, l'auteur du roman avoue que « le seul de leurs livres qui soit bon est celui qui a fait voir le ridicule de tous les autres » (Montesquieu $1964: 135$ ), le traducteur explique au lecteur polonais qu'il s'agit de Don Quichotte (p. 163). Il faut noter que cette référence n'a nullement été suggérée par Montesquieu lui-même.

Ce soin du détail doit sans doute convaincre le lecteur que la version polonaise des Lettres persanes est solide et bien élaborée. Le traducteur le fait croire encore mieux, de manière presque explicite, lorsqu'il mentionne, dans un commentaire à propos de la lettre CXLIII, les problèmes de Montesquieu avec la censure (p. 308). Il dit que ce dernier aurait été contraint d'enlever certains fragments de son roman avant de le confier à son éditeur, lesquels la présente traduction polonaise prétend reconstruire, en offrant au lecteur 161 lettres. Or, le commentaire, loin de relater fidèlement l'histoire de la publication des Lettres persanes du vivant de leur auteur et, surtout, d'indiquer leur version de 1758, rappelle la censure française du XVIII ${ }^{\mathrm{e}}$ siècle pour masquer habilement l'intervention de celle des autorités russes dans la traduction polonaise du roman. En effet, on le voit très bien dans la fameuse lettre LI, où Montesquieu parle de la Russie, de sa civilisation et de son système politique. Il va sans dire que cette lettre a dû attirer une attention toute particulière de la part de la censure russe. Ainsi donc, lorsque le traducteur évoque le fragment du roman, où Montesquieu constate à propos de la Russie qu'" on ne saurait croire combien les femmes moscovites aiment à être battues : elles ne peuvent comprendre qu'elles possèdent le cœur de leur mari s'il ne les bat comme il faut : une conduite opposée, de sa part, est une marque d'indifférence impardonnable » (Montesquieu 1964 : 92), il croit utile, voire nécessaire, d'ajouter une explication selon laquelle ce type de mœurs fut antérieur au tsar Pierre le Grand (p. 101). Il « enrichit » par là le commentaire qu'en a fait Montesquieu qui, en bas de page, a noté de manière peu précise : « Ces mœurs sont changées ». Son intervention ne doit donc que renforcer la propagande russe de l'époque, selon laquelle c'est depuis le règne du fondateur de Saint-Pétersbourg que la Russie se trouve sur une voie de développement civilisateur et de modernisation culturelle constante.

On ne sait pas si le traducteur a fait son commentaire volontairement, ou y a été forcé par la censure russe, tout comme on ignore aussi qui a décidé d'enlever à la lettre LI des fragments entiers. En effet, le lecteur polonais n'apprend rien sur « le climat affreux de la Moscovie », ni que " dès qu'un grand est disgracié, on le relègue en Sibérie » (Montesquieu 1964 : 92). Il ignore également que les Moscovites « ont conservé leurs anciennes coutumes avec d'autant plus d'attachement qu'ils ne croyaient pas qu'il fût possible d'en avoir d'autres », et que « le clergé et les moines n'ont pas moins combattu 
en faveur de leur ignorance » (Montesquieu 1964 : 93). Au portrait du tsar, d'ailleurs appellé ici « empereur », on retire l'information selon laquelle celui-ci est « inquiet et sans cesse agité », et qu'il « erre dans ses vastes états, laissant partout des marques de sa sévérité naturelle » (Montesquieu 1964 : 93). Il ne faut pas, non plus, que l'on se rappelle dans une Pologne sous la domination russe que, déjà au XVIII ${ }^{\mathrm{e}}$ siècle, le tsar tenait à « chercher dans l'Europe d'autres provinces et de nouveaux royaumes » (Montesquieu 1964 : 93). Curieusement, le traducteur polonais, voire la censure russe, n'a pourtant rien contre ce fragment de la lettre LI, où Montesquieu estime que le tsar « est le maître absolu de la vie et de biens de ses sujets, qui sont tous esclaves » (Montesquieu 1964 : 92). Au lieu de trouver ces termes provocants et subversifs, les aurait-on considérés justes et même « politiquement corrects », propres à décrire bien le fondement et le fonctionnement de l'Empire russe ?

Même si l'on ignore aujourd'hui le nom du traducteur polonais des Lettres persanes de 1885, il est intéressant, voire révélateur, de découvrir celui de la maison d'édition, où il a fait paraître son ouvrage. Il s'agit de Przegląd Tygodniowy (Revue hebdomadaire), périodique d'opinion important, publié à Varsovie entre 1866 et 1904, dans le milieu des «positivistes polonais », dont la mission fut de propager les idées de la vie sociale, de la littérature et des beaux-arts, en vue de promulguer la culture et le progrès de la société polonaise (Nofer-Ładyka 1985 : 247). De 1880 à 1903, une fois par mois, ils ambitionnaient de faire accompagner leur hebdomadaire d'un ouvrage littéraire ou scientifique de grande envergure. C'est ainsi que la traduction anonyme des Lettres persanes a vu le jour. Même si l'on voit bien ses défauts, on doit pourtant apprécier le rôle que lui assignaient autant le traducteur que l'éditeur. Tous les deux inscrivaient sans doute le roman de Montesquieu dans leur catalogue d'ouvrages importants, aussi bien pour l'histoire de l'humanité que du point de vue de l'essor de la civilisation. Il est donc capital de remarquer que, selon eux, les Lettres persanes pouvaient jouer un rôle considérable dans les efforts de relever intellectuellement un pays qui, au XIX ${ }^{\mathrm{e}}$ siècle, n'existait même pas sur la carte d'Europe.

Il est presque symbolique que la date de 1918 évoque à la fois la renaissance de la Pologne en tant qu'État libre et indépendant, et la publication d'une nouvelle traduction polonaise des Lettres persanes. Celle-ci sort de la main de Tadeusz Boy-Żeleński, professeur de littérature française, aussi bien son propagateur zélé que le plus célèbre traducteur de ses chefs-d'œuvre. Dans son travail sur le roman de Montesquieu, il traduit, en premier, les deux textes d'auteur qui, depuis 1758, accompagnent habituellement l'ouvrage, aussi bien l'Introduction que les Quelques réflexions sur les « Lettres persanes ». En tant que professeur de littérature, il comprend très bien leur importance et le rôle particulier que leur assignait le romancier. Mieux encore, Boy-Żeleński propose sa propre introduction intitulée Od thumacza (Avis du traducteur), où il présente brièvement (pages I-VI) la vie de Montesquieu, ainsi que le contexte historique et social de l'époque de la Régence. Malheureusement, de tous les ouvrages du philosophe français, il ne mentionne que deux titres : les Considérations sur les causes de la grandeur des Romains et de leur décadence et L'Esprit des lois, en passant sous silence tout le reste de son œuvre. En revanche, il suggère une ressemblance d'esprit et d'intelligence entre Montesquieu et Montaigne, juxtaposition que l'on aime faire en France, à tort ou à raison, encore aujourd'hui (Lacouture 2003). Le traducteur polonais ne cache pas 
que, dans sa brève étude historico-biographique, il s'appuie sur la fameuse monographie qu'Albert Sorel a consacrée à Montesquieu en 1887. Cet emprunt se laisse remarquer surtout dans la manière de voir et de faire voir les Lettres persanes, qualifiées par lui de « quasi romance » (p. IV). Certes, il était trop tôt pour insister alors sur la dimension romanesque de l'ouvrage, rappelée et mise en valeur beaucoup plus tard, seulement dans les années soixante du $\mathrm{XX}^{\mathrm{e}}$ siècle. Il s'agit plutôt de signaler que Sorel et, après lui, Boy-Żeleński (Siemek 2000 : 185) perçoivent le roman dans une perspective prérévolutionnaire des Lumières, ce qui fait que le lecteur ne devrait le comprendre que par rapport à l'avènement de la Révolution de 1789, dont les Lettres persanes sembleraient même annoncer l'arrivée.

Il est intéressant de voir que, dix ans plus tard, Boy-Żeleński agrandit sa propre introduction et $\mathrm{y}$ ajoute un nouveau passage pour une réédition de sa traduction du roman de Montesquieu (celle-ci ne paraîtra pourtant qu'en 1932). C'est ce texte définitif qui, daté «Cracovie 1918 - Varsovie 1928 », accompagne jusqu'aujourd'hui toutes les éditions polonaises des Lettres persanes. La lecture de cette introduction a pourtant de quoi surprendre, car le fragment inséré en 1928 parle paradoxalement moins du roman épistolaire de Montesquieu que de son Esprit des lois. Boy-Żeleński offre même au lecteur polonais un échantillon de la fameuse Invocation aux Muses, texte que Jacob Vernet n'a pas voulu mettre dans la première édition de l'ouvrage en 1748, et montre des fragments de la correspondance entretenue à ce sujet entre le pasteur genevois et l'auteur de L'Esprit des lois (pp. 11-12). Le paradoxe se laisse expliquer, sans qu'il cesse pourtant d'intriguer le lecteur polonais, par le fait que Boy-Żeleński venait juste d'achever sa traduction de l'opus magnum de Montesquieu, qui allait paraître à Varsovie en 1927. Il en a aussi rédigé l'introduction, mais il a dû céder la place au ministre de la Justice, Wacław Makowski, qui voulait proposer sa propre préface, afin de donner plus d'honneur et de majesté à la publication polonaise du célèbre ouvrage de Montesquieu (Matyaszewski 2014 : 42-43). Si le désir du traducteur de sauvegarder son introduction à L'Esprit des lois se laisse donc comprendre facilement, sa décision de la mettre, ne fûtce qu'en partie, dans celle aux Lettres persanes, irrite pourtant jusqu'aujourd'hui à cause de la disproportion de l'ensemble du texte, voire par son caractère hybride manifeste.

Par rapport à l'introduction de 1918, celle de 1928 contient quelques éléments biographiques et littéraires importants. Boy-Żeleński rappelle la personne de Marie-Anne de Bourbon-Condé, dite Mademoiselle de Clermont, et les sentiments d'attachement, sinon d'amour, que Montesquieu aurait éprouvé pour elle lors de ses séjours au château de Chantilly. Il ajoute qu'elle aurait été probablement l'inspiratrice ou la dédicataire du Temple de Gnide de 1725, dont il offre au lecteur polonais la traduction d'un petit fragment (pp. 9-10). Mieux encore, il évoque les salons parisiens de Madame de Tencin, de Madame de Lambert et de Madame du Deffand (en oubliant pourtant celui de Madame Geoffrin), pour rappeler la présence de Montesquieu dans différents milieux mondains de la capitale (p. 9). Le traducteur polonais tient surtout à faire comprendre les Lettres persanes à travers le rôle capital que jouaient les femmes dans la vie intellectuelle de la France du XVIII ${ }^{\mathrm{e}}$ siècle. Sans abandonner sa vision pré-révolutionnaire des Lumières qu'il exprime en 1918, il en conçoit une autre dix ans plus tard, pour faire lire le roman de Montesquieu selon son optique d'historien de la littérature. Non que soutenir le rôle important des femmes et de leurs salons dans l'essor de la culture au XVIII ${ }^{\mathrm{e}}$ siècle 
soit erroné ou abusif, mais la vision qu'en propose Boy-Żeleński est pourtant lourde de fausses interprétations de sa part. Il suggère ouvertement que Montesquieu aurait rédigé son roman pour l'adresser aux femmes et, qui pis est, l'écrivain n'y aurait pas négligé, en esprit pragmatique, la position sociale puissante de ses lectrices, chez qui il aurait cherché protection (pp. 8-10). Cette vision singulière de l'origine des Lettres persanes se laisse renforcer encore mieux par l'histoire évoquée plus haut à propos du Temple de Gnide et de la relation supposée entre Montesquieu et Mademoiselle de Clermont.

Le paradoxe du traducteur polonais, pour ne pas dire son interprétation surprenante des faits, réside aussi ailleurs. D'un côté, Boy-Żeleński propose sa vision des Lettres persanes presque en féministe, en soutenant, d'ailleurs avec beaucoup de justesse, la position particulière des femmes dans la vie intellectuelle en France à l'époque des Lumières. De l'autre, au plus grand étonnement du lecteur, il a pourtant l'air de douter sérieusement de leur intelligence. Lorsqu'il insiste, toujours avec raison, sur la clarté et la précision du style de Montesquieu, il en déduit explicitement qu'elles résulteraient de son désir de se faire mieux comprendre par les femmes et d'adapter ainsi la forme de son ouvrage à leur niveau intellectuel (p. 10). Autrement dit, la netteté du style de l'écrivain serait forcée et, pour ainsi dire, accommodée à la manière dont les femmes savaient lire au XVIII ${ }^{\mathrm{e}}$ siècle. Selon une telle optique, celles-ci, quoique éclairées, auraient eu tout de même besoin d'aide de la part de l'auteur masculin qui, quant à lui, aurait simplifié son propre texte avant de le faire passer à ses lectrices, afin de leur rendre la lecture aussi bien agréable que, surtout, compréhensible. Boy-Żeleński croit fermement et le constate de front que Les Lettres persanes, de même que L'Esprit des lois, en seraient la preuve évidente (pp. 10 et 13). Ses propos semblent autant donner une image peu positive du niveau intellectuel des femmes des Lumières que, sans doute involontairement, mettre en cause le talent de Montesquieu ou, du moins, ridiculiser sa méthode de travail.

C'est ainsi que s'achève la longue histoire des traductions polonaises des Lettres persanes. Elle embrasse trois siècles et trois périodes différentes de l'histoire de la Pologne. La traduction de quelques lettres dans Monitor, ainsi que celle de tout le roman, faite en pionnier par Tomasz Kajetan Węgierski, ont lieu dans une Pologne en déclin qui, à l'époque des Lumières, cherche pourtant à se réformer et à arrêter la catastrophe inévitable de l'État. La traduction suivante, anonyme, paraît au XIX ${ }^{\mathrm{e}}$ siècle, dans une Varsovie occupée par les Russes, quand la Pologne n'existe pas sur la carte géopolitique d'Europe. Tadeusz Boy-Żeleński donne sa traduction juste à la fin de la Grande Guerre, au moment, où la Pologne revient dans la famille des pays libres et indépendants. On pourrait dire que toutes ces traductions du roman de Montesquieu paraissent à chaque fois dans des contextes historiques importants pour la Pologne. Coïncidence de faits, ou bien la preuve que, depuis le temps de Stanisław Konarski, les Polonais voient toujours dans les Lettres persanes plus qu'un simple roman épistolaire? 


\section{BIBLIOGRAPHIE}

AleKSANDROwSKa Elżbieta, 1988, Montesquieu i d'Alembert na łamach monitorowych: z warsztatu bibliografa „Monitora”, Pamiętnik Literacki 79/3 : 155-169.

Konarski Stanisław, 1760, O skutecznym rad sposobie, albo o utrzymywaniu ordynaryjnych seymów, Warszawa : XX Scholarum Piarum, vol. I.

Lacouture Jean, 2003, Montesquieu. Les vendanges de la liberté, Paris : Éditions du Seuil.

Lukowski Jerzy, 2001, L'influence de 1' « Esprit des lois » sur la pensée politique en Pologne au XVIII siècle, Cahiers Montesquieu $6: 49-59$.

Matyaszewski Paweł, 2013, Traduire Montesquieu en Pologne au XVIII' siècle. Étude chronologique, Revue française d'histoire du livre 134 : 159-180.

Matyaszewski Paweł, 2014, Deux traductions polonaises de "l'Esprit des Lois 》 de Montesquieu : de Mateusz Czarnek (1777) à Tadeusz Boy-Żeleński (1927), (in:) Territoires comparatistes. Mélanges offerts à Zbigniew Naliwajek, Anna Opiela (red.), Warszawa : Wydawnictwa Uniwersytetu Warszawskiego, 36-44.

Matyaszewski Paweł, 2018, Monteskiusz w Polsce. Wczoraj i dziś, Warszawa : Wydawnictwa Uniwersytetu Warszawskiego, Instytut Monteskiusza w Krakowie.

Monitor, 1767, 94, 25 XI.

Monitor, 1769, 54, 8 VII.

MoNTESQUIEU Charles-Louis de, 1778, Listy perskie, przettómaczone z francuskiego, Drezno : M. Gröll (vol. I, listy I-LXXVI) ; 1778, Kontynuacja Listów perskich P. de Montesquieu, przettómaczona z francuskiego, Drezno : M. Gröll (vol. II, listy LXXVII-CLXI). Traduction : Tomasz Kajetan Węgierski.

Montesquieu Charles-Louis de, 1885, Listy perskie, przeklad z francuskiego M.W., Warszawa : Wydawnictwo „Przeglądu Tygodniowego”. Traduction anonyme.

Montesquieu Charles-Louis de, 1918, Listy perskie, Kraków : G. Gebethner i Spółka. Traduction : Tadeusz Boy-Żeleński.

MontesQuieu Charles-Louis de, 1932, Listy perskie, Wydanie Nowe, Warszawa : Arct S.A. Traduction : Tadeusz Boy-Żeleński.

Montesquieu Charles-Louis de, 1964, Lettres persanes, Paris : Garnier-Flammarion.

Nofer-Ładyka Alina, 1985, Przegląd Tygodniowy Życia Spotecznego, Literatury i Sztuk Pięknych, (in:) Literatura polska. Przewodnik encyklopedyczny, Cz. Hernas (red.), Warszawa : PWN, t. II, 247.

SIEMEK Andrzej, 2000, Wokót przekładów Boya: tlumaczenie jako wizja obowiązowa, (in:) Spotkania $z$ dawna literatura francuska, K. Dybeł, B. Marczuk (red.), Kraków : Wydawnictwo Uniwersytetu Jagiellońskiego, 183-188.

SmoleŃski Władysław, 1927, Monteskiusz w Polsce wieku XVIII, Warszawa : Instytut Popierania Sztuki.

VolpilhaC-Auger Catherine, 2017, Montesquieu, Paris : Éditions Gallimard. 